\title{
Analysis of Neuropsychiatric Symptoms in Patients with Alzheimer's Disease Using Quantitative EEG and sLORETA
}

\author{
Yong S. Shim ${ }^{a}$ Hae-Eun Shin ${ }^{b}$ \\ ${ }^{a}$ Department of Neurology, The Catholic University of Korea Eunpyeong St. Mary's Hospital, Seoul, South Korea; \\ ${ }^{b}$ Department of Neurology, The Catholic University of Korea Bucheon St. Mary's Hospital, Bucheon, South Korea
}

\section{Keywords}

Neuropsychiatric symptoms · Alzheimer's disease ·

Electroencephalography

\begin{abstract}
Objective: The electrocortical activities associated with the neuropsychiatric symptoms (NPSs) of Alzheimer's disease (AD) were investigated using frequency-domain electroencephalography (EEG) spectral source analysis, and the potential electrocortical indices identified. Materials and Methods: Scalp EEG data were obtained from 51 patients with AD to investigate the presence of four NPS subdomains, hyperactivity, psychosis, affective symptoms, and apathy. EEG power spectra and the standardized low-resolution brain electromagnetic tomography (sLORETA)-localized EEG cortical sources were compared between the groups with and without the four NPS subdomains in eight frequency bands: 1-4, 4-8, 8-10, 10-12, 12-18, 18-20, 20-30, and 30$45 \mathrm{~Hz}$. Results: The power spectral analysis of EEG data showed that AD patients with psychosis had lower values at
\end{abstract}

karger@karger.com www.karger.com/ndd

Karger $\frac{1}{6}$

GOPEN ACCESS
(C) 2020 The Author(s)

Published by S. Karger AG, Basel

This article is licensed under the Creative Commons AttributionNonCommercial-NoDerivatives 4.0 International License (CC BY NC-ND) (http://www.karger.com/Services/OpenAccessLicense) Usage and distribution for commercial purposes as well as any distribution of modified material requires written permission. the $a_{2}$-band in most areas. In patients with apathy, the $\theta$-to- $\beta$ power ratio showed a greater activity over the frontal and central regions. The cortical source analysis using sLORETA revealed that patients with psychosis showed decreased values in the $a_{2}$-band and patients with apathy showed higher $\delta$-values, especially in the right frontal and temporal regions. Conclusion: The results of the present study showed that both classical EEG spectral and EEG source analysis could differentiate patients with and without NPSs, especially psychosis and apathy subdomains. Spectral and sLORETA analyses provided information helpful for a better characterization in patients with NPSs.

(c) 2020 The Author(s)

Published by S. Karger AG, Basel

\section{Introduction}

Alzheimer's disease (AD) is a progressive neurodegenerative disorder that usually starts slowly and gradually worsens over time. Episodic memory loss is one of the earliest manifestations and is accompanied by word-find- 
ing difficulty, visuospatial dysfunction, and executive function impairment $[1,2]$. In addition to the cognitive symptoms, various neuropsychiatric symptoms (NPSs) emerge during the disease course, including delusions, depression, agitation, sleep disturbances, and eating problems, which can cause significant burden and stress to caregivers [3-6]. However, few therapies exist for the treatment of NPSs in dementia to date [7].

For many years, electroencephalography (EEG) has been used in the field of dementia as a noninvasive, less expensive, and easily available diagnostic and research method [8]. In previous studies, patients with AD exhibited slow EEG waves, reduced complexity of EEG signals, and loss in EEG synchrony [9]. Although visual inspection of EEG remains useful in clinical practice, one of the most common methods in the research field is quantitative EEG (qEEG) analysis $[8,10,11]$. In numerous studies, $\mathrm{AD}$ and mild cognitive impairment were associated with an increased power in low frequencies (e.g., $\delta$ - and $\theta$-bands), and a decreased power in higher frequencies ( $\alpha$ - and $\beta$-bands) [11]. In addition, increased global slowto-fast power ratios were strongly correlated with $\mathrm{AD}$ progression [12-14].

Currently, few studies have reported on the association of EEG with the NPSs in patients with AD [15]. In a study on EEG complexity, identifying the neuropsychiatric correlates provided insight into how brain-specific regional activity is associated with these symptoms; disinhibition was associated with frontal EEG complexities, depression and anxiety were correlated with temporal and parietal complexity, and apathy, aberrant behavior, and sleep changes were correlated with parieto-occipital EEG changes [15]. NPSs can always be present during the disease course; however, specific symptoms are more common at different stages. Almost all symptoms worsen with disease severity; however, certain symptoms such as delusions, agitation, and apathy tend to become much more prevalent [16]. Evaluation of the frequency and region-specific qEEG changes associated with NPSs based on qEEG and brain structural change would be informative.

In the present study, the qEEG of patients with $\mathrm{AD}$ was evaluated to investigate the presence of NPSs such as hyperactivity, psychosis, affective symptoms, and apathy to determine whether qEEG frequencies and spectral powers differ by scalp region in patients with $\mathrm{AD}$. Based on the hypothesis that NPSs can provide insight into possible relationships between clinical features and their underlying causal and/or risk factors, different therapeutic strategies targeting subsyndromes may be more effective.

\section{Materials and Methods}

\section{Participants}

The study group included 51 patients with $\mathrm{AD}$ (34 females, 17 males) who were recruited from the Dementia Clinic at the Catholic University of Korea, Bucheon St. Mary's Hospital, from January 2018 to December 2018. The diagnosis for AD was based on the criteria of the National Institute of Neurological and Communicative Disorders and the Stroke/Alzheimer's Disease and Related Disorders Association [17]. All patients received neurological examinations, laboratory tests, EEG monitoring, and neuroimaging evaluation during the diagnostic process. Retrospective analysis of the patients' clinical and EEG data was approved by the Institutional Review Board of the Catholic University of Korea, Bucheon St. Mary's Hospital. Subjects provided informed consent. Patients who had other conditions that might cause cognitive impairment, such as vascular dementia, Parkinson's disease, hypothyroidism, vitamin $B_{12}$ deficiency, syphilis, and history of major psychiatric illness (e.g., major depression, bipolar disorder, or schizophrenia) were excluded.

The severity of dementia was assessed using the Clinical Dementia Rating (CDR) scale [18], and general cognition was evaluated using the Mini-Mental State Examination (MMSE) [19]. NPSs were evaluated using the Neuropsychiatric Inventory (NPI) [3], and a score $>3$ was considered to indicate the presence of clinically relevant symptoms [20-24]. The 12 NPI items were classified into four subsyndromes, corresponding to hyperactivity (agitation, disinhibition, irritability, aberrant motor behavior), psychosis (delusions, hallucinations, night-time behavior disturbances), affective behaviors (depression, anxiety), and apathy (apathy, appetite and eating abnormalities) [25].

Patients who underwent qEEG and did not show an EEG abnormality were included in the present study. An EEG abnormality was defined as asymmetry of background activity, continuous $\theta$-range slow waves, generalized or focal $\delta$-range slow waves, or epileptiform discharges. Patients taking cholinesterase inhibitors, benzodiazepines, or antidepressants that could influence EEG rhythms were excluded [26]. There was no one taking antiepileptic drugs in the study participants.

\section{EEG Recording}

All participants received a routine EEG (COMET plus EEG, Grass Technologies Inc., West Warwick, RI, USA) in a specialized examination room as a part of the initial assessment before starting any specific AD therapy (e.g., acetylcholinesterase inhibitors). Procedures were performed on weekdays between 9 a.m. and 5 p.m.

EEG was recorded under waking-rest conditions (eyes closed) from 19 scalp electrodes positioned based on the International 1020 System (Fp1, Fp2, F7, F3, Fz, F4, F8, T7, C3, Cz, C4, T8, P7, P3, $\mathrm{Pz}, \mathrm{P} 4, \mathrm{P} 8, \mathrm{O} 1$, and O2) with linked ear reference, $256 \mathrm{~Hz}$ sampling rate, high-pass filter of $1 \mathrm{~Hz}$, low-pass filter of $70 \mathrm{~Hz}$, notch filter of $60 \mathrm{~Hz}$, and impedance below $5 \mathrm{k} \Omega$. Activation maneuvers, including opening of eyes, hyperventilation, or photic stimulation were performed under instruction from technicians. Vigilance was monitored by the EEG technician who alerted patients when signs of drowsiness appeared in the tracings; 9-12 artifact-free 4-s epochs were finally obtained per patient. One independent investigator blinded to the diagnosis of participants visually confirmed the EEG segments were acceptable for further analyses. 


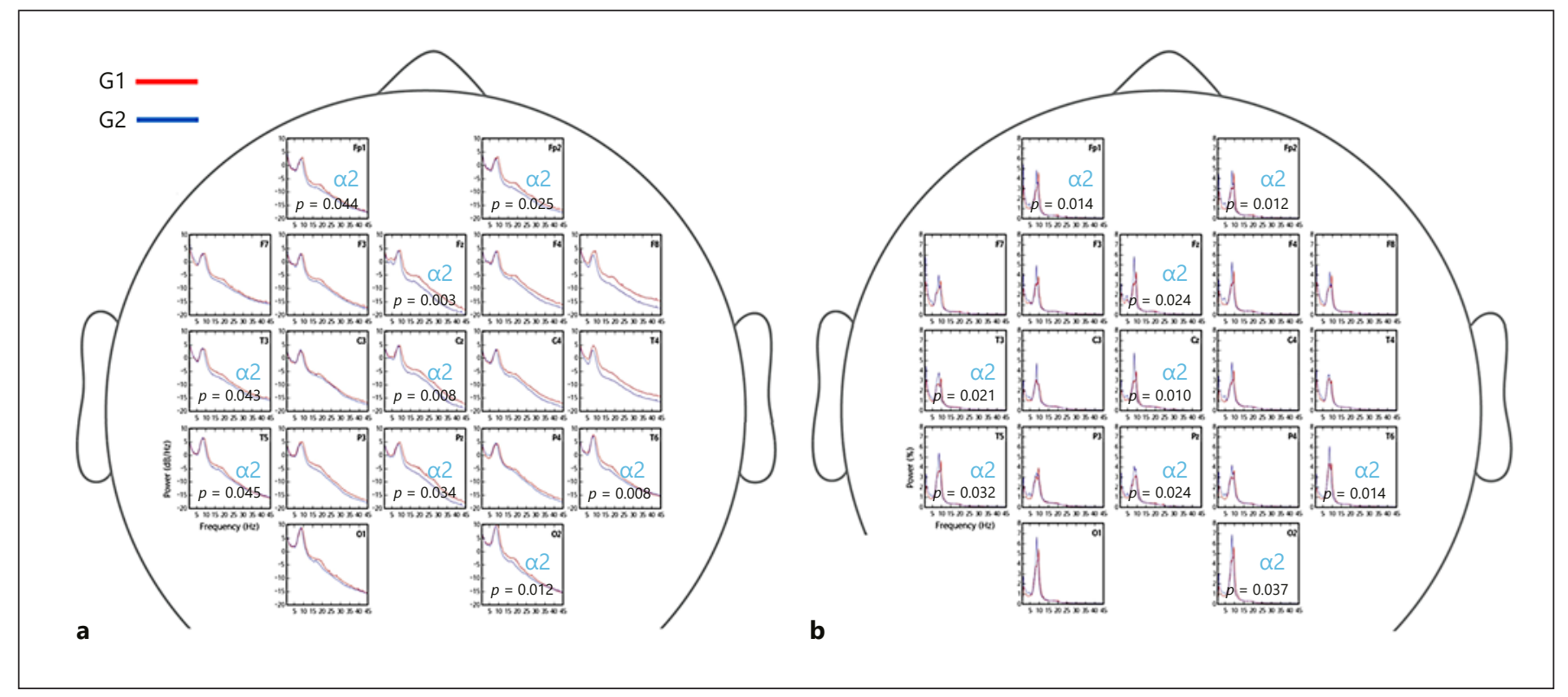

Fig. 1. Absolute (a) and relative (b) electroencephalogram power spectra for each of the 19 channels in groups without (G1) and with (G2) psychosis. Blue letters indicate decreased power in G2.

\section{EEG Analysis}

For preprocessing steps, the EEG data were high-pass filtered offline above $1 \mathrm{~Hz}$, low-pass filtered below $45 \mathrm{~Hz}$, and recomputed to the common average reference. Transient artifacts were rejected with a visual inspection, and stationary artifact components originating from eye movement, muscle tensions, or heartbeat were removed using adaptive mixture independent component analysis [27].

Following the removal of artifacts from the EEG signals, sensor level analysis using spectopo function based on EEGLAB [20] was performed in the following eight spectral bands: $\delta(1-4 \mathrm{~Hz}), \theta(4-8$ $\mathrm{Hz}), \alpha_{1}(8-10 \mathrm{~Hz}), \alpha_{2}(10-12 \mathrm{~Hz}), \beta_{1}(12-15 \mathrm{~Hz}), \beta_{2}(15-20 \mathrm{~Hz}), \beta_{3}$ $(20-30 \mathrm{~Hz})$, and $\gamma(30-45 \mathrm{~Hz})$. In the power spectral density 2 -dimensional map, topomaps of absolute and relative power in $1-\mathrm{Hz}$ bins $(1-45 \mathrm{~Hz})$ as well as each frequency band are presented. Absolute power is the sum of the component powers for each frequency band. Relative power is the absolute power in a specific frequency band divided by the total power. This feature provides absolute and relative power based on five brain regions (frontal, temporal, central, parietal, and occipital). The power spectra for each of the 19 channels are shown in the power spectral density spectrum, which presents the amplitude of power in units of microvolts squared per hertz or decibels per hertz to obtain the frequency characteristics of the $\alpha$-band or $\beta$-band, respectively. Furthermore, the following band power ratios were calculated: $\theta$-to- $\alpha$ (TAR), $\delta$-to- $\alpha$ (DAR), and $\theta$-to- $\beta$ (TBR). Source reconstructions were performed using standardized low-resolution brain electromagnetic tomography (sLORETA) plugin [21] using a Colin 27 Head model [22] with 68 regions of interest segmentations based on the Desikan and Killiany atlas [23]. All preprocessing steps, denoising using adaptive mixture independent component analysis, sensor level feature extractions, and source level feature extractions were performed on iSyncBrain ${ }^{\circledR}[24]$.

\section{Statistical Analysis}

For the four NPSs (hyperactivity, psychosis, affective behaviors, and apathy), clinical characteristics and qEEG analyses were compared between patients with and without NPSs using the Mann-Whitney U test. Statistical significance was accepted for a $p$ value $<0.05$ and performed automatically using the iSyncBrain ${ }^{\circledR}$ [24] program.

\section{Results}

\section{Clinical Characteristics}

A total of 51 patients (17 males, 34 females) with AD were analyzed in the present study. The clinical characteristics were as follows: mean age $77.73 \pm 7.28$ years; duration of education $4.44 \pm 3.90$ years; MMSE score 16.36 \pm 6.43; CDR score $1.16 \pm 0.66$, range 0.5-3.0; CDR sum of boxes score $6.01 \pm 3.83$, range $0-16$.

Differences in age, sex, duration of education, MMSE score, CDR score, and CDR sum of boxes score were not observed between groups with and without the four NPSs (hyperactivity, psychosis, affective symptoms, and apathy), except for the distributions of sex between groups with and without affective symptoms ( 9 males: 28 females vs. 8 males: 6 females, $p=0.028)$. Although duration of education of patients was low, below 6 years, there was no difference between groups with and without the NPSs. 


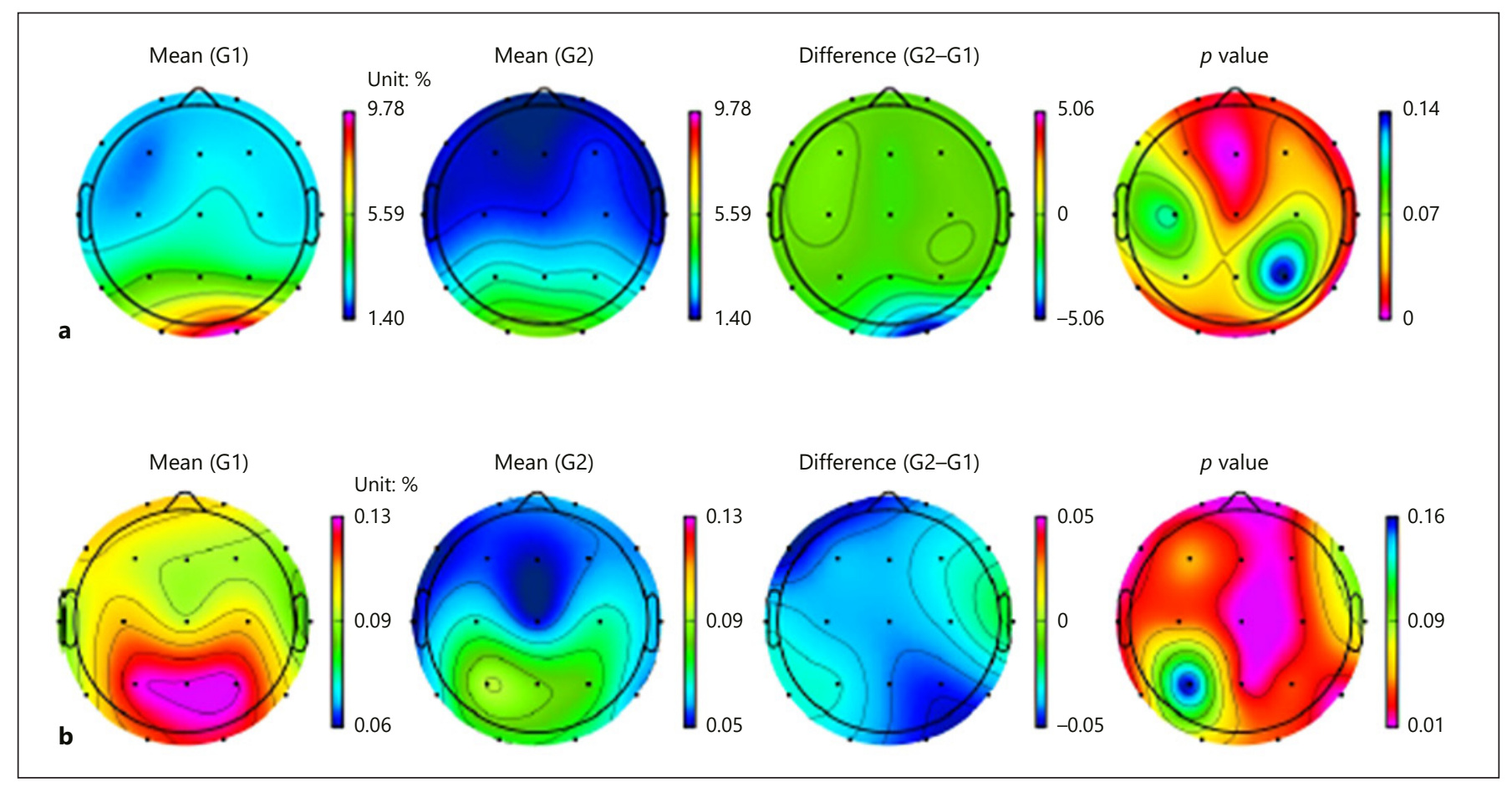

Fig. 2. Scalp topographies of absolute (a) and relative (b) electroencephalogram power differences between groups with (G2) and without (G1) psychosis in the $\alpha_{2}$-frequency band.

\section{Power Spectral Analysis}

Figure 1 shows the absolute and relative EEG power spectra for each of the 19 channels in groups without (G1) and with (G2) psychosis. Blue letters indicate decreased power in G2. Differences between groups with and without hyperactivity, affective symptoms, and apathy were not observed. The EEG power spectrum had lower values at the $\alpha_{2}(10.0-12.0 \mathrm{~Hz})$ frequency band in the channels of $\mathrm{Fp} 1, \mathrm{Fp} 2, \mathrm{Fz}, \mathrm{T} 3, \mathrm{Cz}, \mathrm{T} 5$, and $\mathrm{Pz}$ in $\mathrm{AD}$ patients with psychosis compared with patients without psychosis. This result was further confirmed by the scalp topographies of EEG power differences between groups with and without psychosis in the $\alpha_{2}$-frequency band (Fig. 2). Patients with psychosis showed decreased power of the $\alpha_{2^{-}}$ band in frontal $\left(1.580 \pm 1.395\right.$ vs. $2.590 \pm 1.897 \mu \mathrm{V}^{2}, p=$ $0.016)$, central $\left(1.915 \pm 1.917\right.$ vs. $2.838 \pm 2.354 \mu \mathrm{V}^{2}, p=$ $0.044)$, and temporal $\left(2.219 \pm 1.787\right.$ vs. $3.480 \pm 1.962 \mu \mathrm{V}^{2}$, $p=0.009)$ areas. Over most scalp locations, the average power in $\alpha_{2}(10-12 \mathrm{~Hz})$ largely decreased in patients with psychosis.

Figure 3 shows power ratios (TBR, TAR, and DAR) between groups with and without apathy. Patients with apathy had increased TBR values in frontal (3.634 \pm 2.568 vs. $3.197 \pm 4.266 \mathrm{AU}, p=0.032)$ and central $(2.772 \pm 2.061$ vs. $2.799 \pm 3.567 \mathrm{AU}, p=0.030)$ areas. Scalp topographies of TBR showed prominent differences between patients with and without apathy over the frontal and central regions. Patients with apathy had a greater slow-to-fastwave power ratio. A further comparison between groups with and without apathy based on the Mann-Whitney U test did not show significance when TAR or DAR was used as the power ratio parameter.

Results of absolute power spectrum are usually similar to relative power measure. However, in the present study, the results with significance in both the absolute and relative power spectra were presented to attenuate the interindividual variability of the absolute spectral power value [28].

\section{Cortical Source Analysis}

Neuronal sources with significant power changes in 1-4, $4-8,8-10,10-12,12-18,18-30$, and $30-45 \mathrm{~Hz}$ between patients with and without psychosis and apathy were localized in widespread brain regions based on sLORETA (Table 1). Specifically, the significantly decreased source activities included $\alpha_{2}$-wave oscillatory activity in the right transverse 


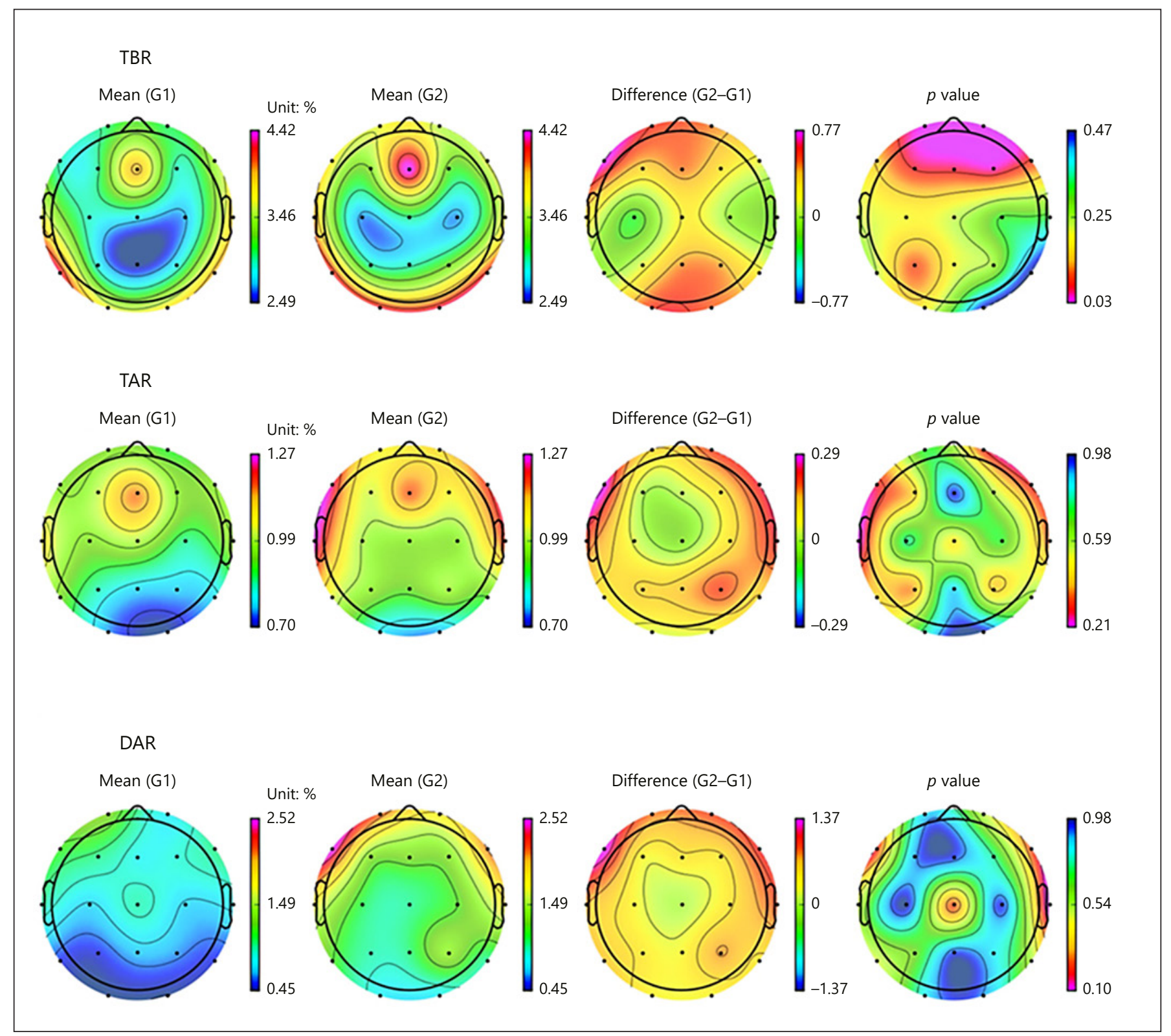

Fig. 3. $\theta$-to- $\beta$ (TBR), $\theta$-to- $\alpha$ (TAR), and $\delta$-to- $\alpha$ (DAR) power ratios between groups with (G2) and without (G1) apathy.

temporal area in patients with psychosis. The significantly increased source activities included $\delta$-waveoscillatory activity in the right superior, middle, and inferior temporal areas and the left parahippocampal regions in patients with apathy. The default mode network showed increased $\delta$-changes in the right temporal (superior and middle) and bilateral entorhinal and parahippocampal areas in patients with apathy. These significant source activities were combined into 68 regions of interest based on anatomic location and frequency band.

\section{Discussion}

The present study was the first in which a power spectral analysis of the four NPSs, hyperactivity, psychosis, affective symptoms, and apathy, was performed in $\mathrm{AD}$ patients. In addition, NPSs at the cortical level were characterized based on frequency-domain EEG source localization using sLORETA.

The power spectral analysis of EEG data showed that AD patients with psychosis had lower values at the $\alpha_{2}$ - 
Table 1. Neuronal sources with significant power changes in $\delta$ and $\alpha_{2}$-frequency bands between patients with and without psychosis and apathy, localized based on standardized low-resolution brain electromagnetic tomography in 68 brain regions of interest

\begin{tabular}{|c|c|c|}
\hline \multirow[t]{2}{*}{ Brain structure } & $\delta$ & $a_{2}$ \\
\hline & L $\quad$ R & L $\quad \mathrm{R}$ \\
\hline
\end{tabular}

Frontal pole

Superior frontal

Rostral middle frontal

Caudal middle frontal

Pars opercularis

Pars orbitalis

Pars triangularis

Medial orbitofrontal

Lateral orbitofrontal

Precentral

Paracentral

Rostral anterior cingulate

Caudal anterior cingulate

Temporal pole

Superior temporal

Middle temporal

Inferior temporal

Transverse temporal

Banks of superior temporal sulcus

Fusiform

Entorhinal

Parahippocampal

Insula

$\triangle$

$\triangle$

$\triangle$

Postcentral

Superior parietal

Inferior parietal

Supramarginal

Precuneus

Posterior cingulate

Isthmus

Lateral occipital

O

Cuneus

Pericalcarine

Lingual

00

The number of voxels with significant power changes is listed separately for the left (L) and right hemispheres (R) for each frequency band. Blue colors indicate decreased power and red colors indicate increased power in groups with neuropsychiatric symptoms. Circles indicate psychosis, triangles indicate apathy. Empty symbols indicate borderline significance.

band in most areas. Decreased $\alpha$ indicates failure to relax and self-warming for external stimulation [29]. Patients with apathy showed abnormal findings in the power ratio where scalp topographies of TBR showed a greater slowto-fast-wave power ratio over the frontal and central regions. TBR is the ratio of $\theta(4-8 \mathrm{~Hz})$ to $\beta(13-21 \mathrm{~Hz})$ pow- er during resting conditions and tends to reflect attentionrelated functions [30, 31]. Increased TBR is associated with increased reward responsiveness, risk taking, and impulsiveness and tends to reflect attentional control functioning and behavioral inhibition processes. Researchers also demonstrated an increased slow-to-fast band power ratio in advanced $\mathrm{AD}$ over all brain regions except the frontal area, which may be caused by contamination of the EEG signal by eye movement [14].

The cortical source analysis of EEG showed the NPSs induced significant changes in source power across different frequency bands in many brain regions. Patients with psychosis showed decreased values in the $\alpha_{2}$-band and patients with apathy showed higher values in $\delta$, especially in the right frontal and temporal regions. In the present study, sLORETA results were similar to the classical spectral analysis, which is consistent with previous reports regarding the LORETA approach in AD [32-34]. The sLORETA results of patients with apathy showed increased $\delta$-power, primarily in the right side and central areas. Both spectral and sLORETA values for $\alpha$-rhythm were relatively preserved over the frontal areas in patients with psychosis compared with patients without psychosis. This finding may indicate the so-called "anteriorization" of $\alpha$-rhythm in $\mathrm{AD}$; in healthy controls, $\alpha$-wave generators are localized in the posterior brain regions while decreased posterior $\alpha$-activity produces a shift of $\alpha$-wave generator to more anterior regions in patients with $\mathrm{AD}$, as previously reported [35-37].

New neuroimaging techniques have been used in recent neurobiological studies to elucidate behaviorally relevant circuits and networks associated with these subsyndromes [38]. Several frontosubcortical circuits, corticocortical networks, and neurotransmitter systems have been proposed as regions and mechanisms underlying NPS-AD. Common to most of these subsyndromes is the broad overlap of regions associated with the salience network (anterior cingulate and insula), mood regulation (amygdala), and motivated behavior (frontal cortex). Physiologically, EEG cortical activity depends on a complex balance among different neurotransmitter systems, primarily cholinergic pathways [39]. $\alpha$-Rhythms are mainly modulated by thalamocortical interactions facilitating or inhibiting the transmission of sensorimotor and cognitive information among subcortical and cortical pathways [40-42]. Therefore, the magnitude reduction of fast cortical rhythms in mild AD is hypothetically associated with impairment of the cholinergic pathway resulting in an abnormal increase in cortical excitation or disinhibition during the resting state. Developing rational thera- 
peutic approaches for NPS-AD will require a better understanding of the underlying etiology, which can be aided with qEEG analysis. Furthermore, qEEG can be useful to predict the effects of treatment for NPSs. The results of the present study are meaningful for the implementation of EEG for the timely and proper treatment based on pathophysiological aspects of the NPSs of dementia although certain limitations remain.

The present study had several limitations. First, pathological or biomarker confirmation was not obtained; however, all the diagnoses were clinically performed with supportive MRI findings. Use of biomarkers such as amyloid positron emission tomography or cerebrospinal fluid can help detect other causes of dementia than AD. Second, the number of participants was relatively small. In addition, EEG activity can be influenced by various factors such as age and the severity of dementia [43, 44]. Further studies with a larger study cohort, analysis of individual NPI items, and adjusted analysis of covariates including age and MMSE can strengthen our results.

In summary, the results of the present study showed that both classical EEG spectral analysis and EEG source analysis can classify patients with and without NPSs including hyperactivity, psychosis, affective symptoms, and apathy. Spectral and sLORETA analyses provided helpful information for a better characterization of qEEG in patients with NPSs. A more accurate analysis could be obtained in further studies if a greater sample size and a combination of spectral and sLORETA analyses with imaging techniques are used.

\section{Acknowledgment}

We would like to thank the research group in the iMediSync Inc., South Korea, which developed source level feature extractions and group statistics functionalities, and applied those to the research data set on iSyncBrain. We would like to thank all their valuable assistances for the analysis and reviewing of the result.

\section{Statement of Ethics}

This retrospective study was performed in accordance with the World Medical Association Declaration of Helsinki and approved by the Institutional Review Board of the Catholic University of Korea, Bucheon St. Mary's Hospital (HC18OESI0114). We confirm that we have read the position of Neurodegenerative Diseases on issues involved in ethical publication and affirm that this work is consistent with the guidelines.

\section{Disclosure Statement}

The authors have no conflicts of interest to declare.

\section{Author Contributions}

Yong S. Shim: concept and design, organization and execution of the study, analysis and interpretation of data, and drafting of the manuscript, review and critique of the manuscript. Hae-Eun Shin: acquisition of data and drafting of the manuscript, review and critique of the manuscript. All authors (Yong S. Shim and Hae-Eun Shin): final approval of the version to be published.

\section{References}

1 Perry RJ, Watson P, Hodges JR. The nature and staging of attention dysfunction in early (minimal and mild) Alzheimer's disease: relationship to episodic and semantic memory impairment. Neuropsychologia. 2000;38(3): 252-71.

2 Baudic S, Barba GD, Thibaudet MC, Smagghe A, Remy P, Traykov L. Executive function deficits in early Alzheimer's disease and their relations with episodic memory. Arch Clin Neuropsychol. 2006 Jan;21(1):15-21.

3 Cummings JL, Mega M, Gray K, RosenbergThompson S, Carusi DA, Gornbein J. The Neuropsychiatric Inventory: comprehensive assessment of psychopathology in dementia. Neurology. 1994 Dec;44(12):2308-14.

4 Devanand DP, Jacobs DM, Tang MX, Del Castillo-Castaneda C, Sano M, Marder K, et al. The course of psychopathologic features in mild to moderate Alzheimer disease. Arch Gen Psychiatry. 1997 Mar;54(3):25763.
5 Donaldson C, Tarrier N, Burns A. The impact of the symptoms of dementia on caregivers. Br J Psychiatry. 1997 Jan;170(1):62-8.

6 Murman DL, Chen Q, Powell MC, Kuo SB, Bradley CJ, Colenda CC. The incremental direct costs associated with behavioral symptoms in AD. Neurology. 2002 Dec;59(11): 1721-9.

7 Lanctôt KL, Amatniek J, Ancoli-Israel S, Arnold SE, Ballard C, Cohen-Mansfield J, et al. Neuropsychiatric signs and symptoms of Alzheimer's disease: new treatment paradigms. Alzheimers Dement (N Y). 2017 Aug;3(3):440-9.

8 Maurer K, Dierks T. Functional imaging procedures in dementias: mapping of EEG and evoked potentials. Acta Neurol Scand Suppl. 1992;139:40-6.

9 Dauwels J, Srinivasan K, Ramasubba Reddy M, Musha T, Vialatte FB, Latchoumane C, et al. Slowing and loss of complexity in Alzheimer's EEG: two sides of the same coin? Int J Alzheimers Dis. 2011 Apr;2011:539621.
10 Brenner RP, Ulrich RF, Spiker DG, Sclabassi RJ, Reynolds CF 3rd, Marin RS, et al. Computerized EEG spectral analysis in elderly normal, demented and depressed subjects. Electroencephalogr Clin Neurophysiol. 1986 Dec;64(6):483-92.

11 Schreiter-Gasser U, Gasser T, Ziegler P. Quantitative EEG analysis in early onset Alzheimer's disease: a controlled study. Electroencephalogr Clin Neurophysiol. 1993 Jan;86(1):15-22.

12 Prinz PN, Vitiello MV. Dominant occipital (alpha) rhythm frequency in early stage Alzheimer's disease and depression. Electroencephalogr Clin Neurophysiol. 1989 Nov;73(5):427-32.

13 Kuskowski MA, Mortimer JA, Morley GK, Malone SM, Okaya AJ. Rate of cognitive decline in Alzheimer's disease is associated with EEG alpha power. Biol Psychiatry. 1993 Apr; 33(8-9):659-62.

14 Bennys K, Rondouin G, Vergnes C, Touchon J. Diagnostic value of quantitative EEG in Alzheimer's disease. Neurophysiol Clin. 2001 Jun;31(3):153-60. 
15 Yang AC, Wang SJ, Lai KL, Tsai CF, Yang CH, Hwang JP, et al. Cognitive and neuropsychiatric correlates of EEG dynamic complexity in patients with Alzheimer's disease. Prog Neuropsychopharmacol Biol Psychiatry. 2013 Dec;47:52-61.

16 Lyketsos CG, Lopez O, Jones B, Fitzpatrick AL, Breitner J, DeKosky S. Prevalence of neuropsychiatric symptoms in dementia and mild cognitive impairment: results from the cardiovascular health study. JAMA. 2002 Sep; 288(12):1475-83.

17 McKhann GM, Knopman DS, Chertkow H, Hyman BT, Jack CR Jr, Kawas CH, et al. The diagnosis of dementia due to Alzheimer's disease: recommendations from the National Institute on Aging-Alzheimer's Association workgroups on diagnostic guidelines for $\mathrm{Al}$ zheimer's disease. Alzheimers Dement. 2011 May;7(3):263-9.

18 Morris JC. The Clinical Dementia Rating (CDR): current version and scoring rules. Neurology. 1993 Nov;43(11):2412-4.

19 Folstein MF, Folstein SE, McHugh PR. "Minimental state". A practical method for grading the cognitive state of patients for the clinician. J Psychiatr Res. 1975 Nov;12(3):189-98.

20 Delorme A, Makeig S. EEGLAB: an open source toolbox for analysis of single-trial EEG dynamics including independent component analysis. J Neurosci Methods. 2004 Mar; 134(1):9-21.

21 Pascual-Marqui RD. Standardized low-resolution brain electromagnetic tomography (sLORETA): technical details. Methods Find Exp Clin Pharmacol. 2002;24 Suppl D:5-12.

22 Holmes CJ, Hoge R, Collins L, Woods R, Toga AW, Evans AC. Enhancement of MR images using registration for signal averaging. J Comput Assist Tomogr. 1998 Mar-Apr;22(2):32433.

23 Desikan RS, Ségonne F, Fischl B, Quinn BT, Dickerson BC, Blacker D, et al. An automated labeling system for subdividing the human cerebral cortex on MRI scans into gyral based regions of interest. Neuroimage. 2006 Jul; 31(3):968-80
24 iSyncBrain. 2019. Retrieved Apr 24, 2019 from: https://isyncbrain.com/research/.

25 Aalten P, Verhey FR, Boziki M, Bullock R, Byrne EJ, Camus V, et al. Neuropsychiatric syndromes in dementia. Results from the European Alzheimer Disease Consortium: part I. Dement Geriatr Cogn Disord. 2007;24(6): 457-63.

26 Babiloni C, Binetti G, Cassarino A, Dal Forno G, Del Percio C, Ferreri F, et al. Sources of cortical rhythms in adults during physiological aging: a multicentric EEG study. Hum Brain Mapp. 2006 Feb;27(2):162-72.

27 Delorme A, Palmer J, Onton J, Oostenveld R, Makeig S. Independent EEG sources are dipolar. PLoS One. 2012;7(2):e30135.

28 Nuwer MR. Quantitative EEG: I. Techniques and problems of frequency analysis and topographic mapping. J Clin Neurophysiol. 1988 Jan;5(1):1-43.

29 Abhang PA, Gawali BW, Mehrotra SC. Chapter 4 - time and frequency analysis. In: $\mathrm{Ab}$ hang PA, Gawali BW, Mehrotra SC, editors. Introduction to EEG- and speech-based emotion recognition. Cambridge: Academic Press; 2016. p. 81-96.

30 Monastra VJ, Lubar JF, Linden M, VanDeusen P, Green G, Wing W, et al. Assessing attention deficit hyperactivity disorder via quantitative electroencephalography: an initial validation study. Neuropsychology. 1999 Jul;13(3):424-33.

31 Demos JN. Getting started with neurofeedback. New York: W.W. Norton; 2005.

32 Babiloni C, Binetti G, Cassetta E, Cerboneschi D, Dal Forno G, Del Percio C, et al. Mapping distributed sources of cortical rhythms in mild Alzheimer's disease. A multicentric EEG study. Neuroimage. 2004 May;22(1):57-67.

33 Babiloni C, Frisoni GB, Pievani M, Toscano L, Del Percio C, Geroldi C, et al. White-matter vascular lesions correlate with alpha EEG sources in mild cognitive impairment. Neuropsychologia. 2008;46(6):1707-20.

34 Babiloni C, Frisoni GB, Pievani M, Vecchio F, Lizio R, Buttiglione M, et al. Hippocampal volume and cortical sources of EEG alpha rhythms in mild cognitive impairment and Alzheimer disease. Neuroimage. 2009 Jan; 44(1):123-35.
35 Ihl R, Dierks T, Martin EM, Froölich L, Maurer $\mathrm{K}$. Topography of the maximum of the amplitude of EEG frequency bands in dementia of the Alzheimer type. Biol Psychiatry. 1996 Mar;39(5):319-25.

36 Claus JJ, Kwa VI, Teunisse S, Walstra GJ, van Gool WA, Koelman JH, et al. Slowing on quantitative spectral EEG is a marker for rate of subsequent cognitive and functional decline in early Alzheimer disease. Alzheimer Dis Assoc Disord. 1998 Sep;12(3):167-74

37 Dierks T, Jelic V, Pascual-Marqui RD, Wahlund $\mathrm{L}$, Julin $\mathrm{P}$, Linden DE, et al. Spatial pattern of cerebral glucose metabolism (PET) correlates with localization of intracerebral EEG-generators in Alzheimer's disease. Clin Neurophysiol. 2000 Oct;111(10):1817-24.

38 Nowrangi MA, Lyketsos CG, Rosenberg PB. Principles and management of neuropsychiatric symptoms in Alzheimer's dementia. Alzheimers Res Ther. 2015 Jan;7(1):12.

39 Selden NR, Gitelman DR, Salamon-Murayama N, Parrish TB, Mesulam MM. Trajectories of cholinergic pathways within the cerebral hemispheres of the human brain. Brain. 1998 Dec;121(Pt 12):2249-57.

40 Steriade M, Llinás RR. The functional states of the thalamus and the associated neuronal interplay. Physiol Rev. 1988 Jul;68(3):649-742.

41 Brunia CH. Neural aspects of anticipatory behavior. Acta Psychol (Amst). 1999 Apr;101(23):213-42.

42 Pfurtscheller G, Lopes da Silva FH. Event-related EEG/MEG synchronization and desynchronization: basic principles. Clin Neurophysiol. 1999 Nov;110(11):1842-57.

43 Pasquier F, Lebert F, Lavenu I, Guillaume B. The clinical picture of frontotemporal dementia: diagnosis and follow-up. Dement Geriatr Cogn Disord. 1999;10 Suppl 1:10-4.

44 Ng TP, Niti M, Chiam PC, Kua EH. Ethnic and educational differences in cognitive test performance on mini-mental state examination in Asians. Am J Geriatr Psychiatry. 2007 Feb;15(2):130-9. 